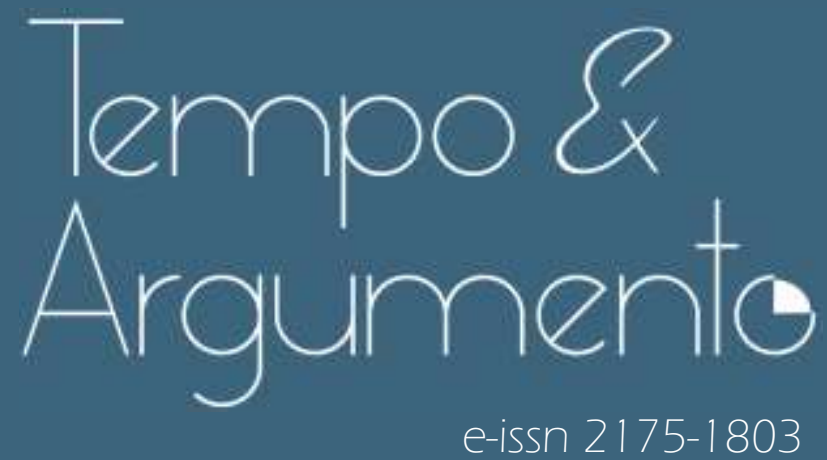

La historia reciente/presente desde una perspectiva de los problemas sociales en la educación de ciudadanías

Miguel Angel Jara

Doctor en Didáctica de las Ciencias Sociales por la Universidad Autónoma de Barcelona. Docente de la Universidad Nacional del Comahue.

Neuquén - ARGENTINA

mianjara@gmail.com

(1) orcid.org/0000-0002-0959-9000

Para citar este articulo (ABNT):

JARA, Miguel Angel. La historia reciente/presente desde una perspectiva de los problemas sociales en la educación de ciudadanías. Tempo e Argumento,

Florianópolis, v. 13, n. 33, e0 108, maio/ago. 2021.

do http://dx.doi.org/10.5965/2175180313332021 e0108

Recebido: 25/02/2021

Aprovado: 16/06/2021 


\title{
La historia reciente/presente desde una perspectiva de los problemas sociales en la educación de ciudadanías
}

\begin{abstract}
Resumen
El presente artículo recoge, con nuevas lecturas, los datos e información que hemos obtenido en nuestros procesos de investigación. Indagar sobre la enseñanza de la historia en la escuela secundaria, fundamentalmente la historia reciente/presente (HRP), nos ha posibilitado comprender la compleja trama entre disciplina, enseñanza, finalidades, formas y contenidos, todo ello en el marco de los cambios producidos a nivel glocal. El escrito dialoga con perspectivas historiográficas, didácticas, sociales, filosóficas, con la intención de pensar en las nuevas configuraciones de una época que desafían y tensionan las prácticas de hacer y enseñar historia. Además, conjuga ideas que contribuyan a profundizar el pensar didácticamente la historia, desde una perspectiva renovada que posibilite abordar la HRP desde los problemas sociales (PS) para la educación de ciudadanías (EC) preocupadas y sensibles por el mundo que habitan.
\end{abstract}

Palabras clave: historia reciente/presente; enseñanza; ciudadanía; problemas sociales.

\section{Recent/present history from a social issue perspective in citizenship education}

\begin{abstract}
This article gathers, using new readings, data and information that we have obtained in our research processes. Addressing history teaching in secondary school, fundamentally recent/present history (RPH), has enabled us to see the complex fabric between discipline, teaching, purposes, forms, and contents, all within the framework of changes produced at the glocal level. The text dialogues with historiographic, didactic, social, philosophical perspectives, in order to think about the new configurations of an era that challenge and stress history making and teaching practices. Also, it combines ideas that contribute to deepening didactic thinking about history, from a renewed perspective that makes it possible to address RPH through social issues (SIS) for citizenship education (CE) concerned with and sensitive about the world that people inhabit.
\end{abstract}

Keywords: recent/present history; teaching; citizenship; social issues.

\section{A história recente/presente sob uma perspectiva dos problemas sociais na educação de cidadanias}

\section{Resumo}

Este artigo reúne, com novas leituras, os dados e as informações que obtivemos em nossos processos de pesquisa. Perguntar sobre o ensino de história na educação secundária, fundamentalmente a história recente/presente (HRP), permite-nos compreender a complexa trama entre disciplina, ensino, finalidades, formas e conteúdos, tudo no marco das mudanças produzidas em nível glocal. O texto dialoga com perspectivas historiográficas, didáticas, sociais, filosóficas, a fim de pensar as novas configurações de uma época que desafiam e tensionam as práticas de fazer e ensinar história. Além disso, reúne ideias que contribuem para aprofundar o pensamento didático sobre a história, sob uma perspectiva renovada que possibilite abordar a HRP por meio dos problemas sociais (PS) para a educação de cidadanias (EC) preocupadas com e sensíveis (a)o mundo que habitam.

Palavras-chave: história recente/presente; ensino; cidadania; problemas sociais. 


\section{Introducción}

Nuestros escritos se configuran en un lugar de la norpatagonia argentina. Revelan las tramas constitutivas de nuestras investigaciones, el oficio de enseñar y de aprender historias, la formación para enseñar historias y el diálogo permanente con otras y otros que tienen las mismas preocupaciones:

- ¿De qué manera contribuimos a producir conocimiento situado para mejorar las prácticas de enseñanza desde la historia reciente/presente?

- ¿Qué problemas sociales abordar en la formación de ciudadanías que habitan el siglo XXI?

Por más de 2 décadas hemos procurado desentrañar, para comprender, las prácticas del profesorado que enseña historia, ciencias sociales y geografía en la escuela secundaria. Hemos aprendido que la historia escolar, en las prácticas situadas del profesorado de la región, es múltiple y plural y que siempre ha estado orientada por finalidades profundamente políticas. Algunas sostenidas en las tradiciones historiográficas, otras en prescripciones curriculares y textos escolares, pero siempre, según la palabra del profesorado, con la clara intención de formar ciudadanías críticas en un vertiginoso mundo que cada vez se presentaba más global.

Las investigaciones realizadas en cada época denotan la dinámica sociocultural y política de un escenario complejo. Procesos epistemológicos, metodológicos, educativos, institucionales, formativos y generacionales reconfiguraron modos de pensar y de accionar en la enseñanza de la historia. El contexto nos ha posibilitado establecer relaciones diversas con nuestro objeto de estudio, de allí que la historiografía, la didáctica de la historia y la metodología de la investigación educativa interactúan para comprender las prácticas de enseñanza y de aprendizaje en los umbrales de este siglo.

La investigación cualitativa crítica, actividad subjetiva que pone en evidencia experiencias y valoraciones, nos indica que, además del involucramiento y compromiso de los profesores que enseñan el pasado reciente, se suma la experiencia vital de ser sujetos históricos, sujetos que toman posiciones frente a temas problemáticos y conflictivos de la dinámica de la realidad social. La educación ciudadana (EC), los derechos humanos y la 
participación ciudadana es, ante todo, cuestión de derechos y convicciones éticopolíticas, de saberes, de valores y donde cada uno de estos criterios educativos se combina en la relación entre enseñanza de la historia y dinámica social (FUNES; JARA, 2017, p. 68).

La historia escolar ha tenido siempre como finalidad la formación ciudadana. Esta finalidad ha estado orientada por decisiones políticas en cada época. Se trata de una formación que ha sido dinámica en su planteamiento curricular, aunque debemos reconocer que con ciertas matrices de larga duración en las prácticas concretas. La enseñanza de la historia en la actualidad ha puesto en tensión los principios de una historia ligada a la identidad nacional. Se trata de una época en la que la historia escolar abre sus miradas a un escenario profundamente político, que disputa sentidos y valores para la democratización de la sociedad, la ciudadanía, los derechos humanos, las identidades, entre otros.

El cambio de perspectiva en la historia enseñada ha permitido incorporar los procesos de la historia reciente/presente (HRP) en tanto son la clave para comprender e interpretar el complejo mundo en el que vivimos. En este sentido, perspectivas y enfoques didácticos críticos han posibilitado incorporar problemas sociales (PS) como organizadores de contenidos históricos escolares. A partir de nuestras investigaciones pretendemos dar cuenta de cómo la HRP se liga profundamente con la EC desde los PS. Por ello recuperamos, con nuevas lecturas, preocupaciones e intereses, aún latentes, para dar cuenta de las relaciones entre la historia y la enseñanza, las finalidades, la comunicación de conocimiento social y las prácticas de enseñanza de la HRP con relación a la EC, atendiendo a PS de esta época.

En el primer apartado damos cuenta de un proceso de delimitación y denominación de un campo de estudio -HRP- que no solo estuvo signado por debates historiográficos, sino por decisiones del profesorado. Ambas cuestiones se cruzan entre lo académico y lo político en cuanto a la función social de la historia y ponen en tensión prácticas y habilitan desafíos como nuevas oportunidades epistemológicas. 
La historia reciente/presente desde una perspectiva de los problemas sociales en la educación de ciudadanías

En el segundo apartado establecemos relaciones entre las distintas finalidades de la EC desde una perspectiva y un enfoque centrados en PS, entendiendo que estos últimos son los organizadores de temporalidades móviles, dinámicas y flexibles de los procesos indeterminados que caracterizan a la HRP.

Finalmente, concluimos con algunas notas que posibiliten seguir entretejiendo la HRP con los PS para una EC en tiempos de incertidumbre. Un desafío epocal para construir otras narrativas con otras temporalidades que contribuyan a pensar en el complejo mundo que habitamos. El debate queda abierto para construcciones colectivas.

\section{Historias y enseñanzas: delimitación y denominación de un tiempo histórico}

Cuando iniciamos nuestras investigaciones ${ }^{1}$ para comprender e interpretar qué historias se enseñan en las aulas de escuelas secundarias de 2 provincias de nuestra región patagónica -Neuquén y Río Negro- y con la perspectiva de construir conocimiento didáctico sobre la historia escolar, específicamente de la HRP, nos encontramos con un interesante debate que se disputaba sentidos y significaciones sobre la posibilidad de una nueva historia, a partir de los cambios acelerados y las reconfiguraciones que a nivel global se venían produciendo e interpelaban fuertemente el oficio de hacer y enseñar historias.

Los inicios de nuestras investigaciones coinciden con el cambio de milenio, pero recogen un debate fructífero en el campo historiográfico -también de otras disciplinas del campo de ciencias las sociales y humanas- sobre ciertos retornos de viejas vigentes y conocidas discusiones -objetividad, protagonistas, fuentes,

\footnotetext{
'Los 5 proyectos de investigación (PI) que hemos llevado a cabo son:

- Durante el periodo 2002-2005: "Estudiantes y enseñanza: el caso de la historia y la geografía" (PI C051);

- Durante 2006-2009: “Lo reciente/presente en la enseñanza de la historia” (PI C068);

- Entre 2010-2012: "Profesores y enseñanza de la HRP" (PI C089) -y ligado a este tema, pero desde una perspectiva de la cultura digital, abordamos durante 2013-2016: "Enseñanza y aprendizaje de las ciencias sociales y de la historia en la cultura digital” (PI C106)-; y

- Finalmente, durante 2017-2020: "El aprendizaje escolar de las ciencias sociales en la cultura digital" (P.I C129).

Todos fueron aprobados y financiados por la Secretaría de Ciencia y Técnica (SECYT) de la Universidad Nacional del Comahue.

Los 4 primeros fueron dirigidos por la Dra. Graciela Funes.
} 
periodizaciones, entre otros- iniciadas en la década de 1970. También de aquellas nuevas que pusieron en tensión los modos estructurales de comprensión, análisis y explicación del pasado. En este viraje, las experiencias y la visibilización de las ausencias en los grandes relatos, cobran relevancia desde las memorias y subjetividades individuales y colectivas, es así como historia-memoria-política se imbrican para dar cuenta de los PS actuales latentes, controversiales y sensibles en escenarios complejos.

Comprendimos que no solo se trataba de un debate por asignarle una denominación o delimitación temporal a los procesos de la HRP, sino de un debate que ponía en evidencia supuestos teóricos y metodológicos poco cuestionados hasta entonces en el campo historiográfico y, por ende, interpelaba perspectivas y enfoques propios del campo de la didáctica de la historia. Se trataba de pensar en otras narrativas, inclusoras y con sentidos de lo plural y heterogéneo.

Entre varios escritos, que han sido producidos en el marco de nuestras investigaciones, recupero el más reciente. Con Graciela Funes (2020) planteamos algunos semblantes del debate que se generó sobre la diversos aspectos -la memoria, la temporalidad, lo político, la subjetividad o las narrativas, entre otrosque marcaron inicialmente la agenda a principios del siglo pasado. En el debate historiográfico, la denominación de esta época invirtió un gran esfuerzo intelectual por delimitar temporalmente los acontecimientos, sucesos o fenómenos vertiginosos que configuraban sociedades, políticas, economías y manifestaciones culturales (JARA; FUNES, 2020, p. 35).

En este contexto nos sumergimos en la búsqueda de respuestas a la crisis que atraviesa la historia como ciencia, para poder pensar didácticamente este problema epistemológico. Diversas posturas historiográficas plantean la necesidad de construir una historia del presente como un nuevo enfoque o perspectiva, asociada a una nueva concepción de temporalidad. Por citar solo algunos de los autores, por ejemplo, Aròstegui (1998) abandona el término reciente por considerarlo demasiado vinculado a lo instantáneo. Propone usar el término presente porque pone el énfasis en la cuestión del tiempo y su concepción. Dice el autor: 
Realidades sociales "recientes", había dicho yo años antes en vez de "actuales" o "presentes" como diría ahora y esa denominación no fue enteramente rechazada por otros autores como alternativa a la de "tiempo presente", que nos parecía excesivamente francesa. "Reciente" sin embargo, no nos parece en momento que recoja ya con claridad el contenido de lo que está en desarrollo, de lo que se desenvuelve de forma actual y determinada la trayectoria de las generaciones vivas. La preocupación por el tiempo presente, por la historia de lo coetáneo en otras palabras no es nueva para nosotros (ARÒSTEGUI, 1998, p. 31-32).

Si bien reconoce que faltan definiciones más claras y análisis de conceptos, intenta diferenciar su posición de aquellos que utilizan este tramo de la historia como un apéndice cronológico de la historia contemporánea. Aròstegui (1998) sostiene que la historia presente necesita una nueva argumentación que dé cuenta de una nueva concepción de la experiencia histórica. Su esfuerzo se centra en contornear, conceptualizar el tiempo presente como una parte esencial de la temporalidad histórica.

Pero no debe identificarse esa historia [habla de lo que él denomina Historia del Mundo actual como asignatura] con la del tiempo presente. La razón primera y primordial es sencilla: la historia presente es incondicionalmente historia viva, no historia reciente, ni inmediata, por mucho que tenga de estas características o connotaciones. Por mucho que se acerque a nuestro propio tiempo [...] tiempo presente es el que se confunde, el que equivale, al tiempo vivido para cada uno de los sujetos históricos. Tiempo presente es para cada cual únicamente el tiempo vivido (ARÒSTEGUI, 1998, p. 34-38).

Frente a estos argumentos, el presente como realidad sociohistórica "es aquel que cabe en la memoria de la gente, moldeado entre su campo de experiencia y su horizonte de expectativa" (ARÒSTEGUI, 1997, p. 40). La propuesta de Aróstegui (1997) pone de relieve la proximidad al sujeto y su carácter de inacabado. Enfatiza en que la construcción y la explicación de la historia de cada época son reveladas desde la perspectiva de los propios protagonistas, de allí que la historia del presente es una categoría histórica y para nada un periodo concreto.

La perspectiva de la historia del presente como categoría histórica, en el proceso de nuestras investigaciones, vino a enriquecer nuestro enfoque, en tanto no se reduce a una simple delimitación cronológica de los fenómenos históricos 
y abre camino a otros posibles trayectos interpretativos a partir de que se constituye en categoría de análisis. Es decir, se trata de una nueva forma de entender el presente y no simplemente de una nueva fase de la historia contemporánea. Pensar en esta posibilidad permite comprender los procesos actuales en su carácter de inacabados, las formas y lógicas de su configuración. En la enseñanza permite incorporar problemas vivos, candentes, conflictivos, relevantes que, siempre controversiales, contribuyen a la construcción de la conciencia y el pensamiento histórico, en una nueva reconexión entre el pasado y el presente para construir los futuros posibles y deseables.

Entre otros aportes que sostienen la perspectiva de una historia del presente, desde un punto de vista filosófico, Mudrovcic (2000, p. 22) la define como "aquel lapso del pasado que tiene por objeto acontecimientos o fenómenos sociales que constituyen recuerdos de al menos una de las tres generaciones que comparten un mismo presente histórico". La autora sostiene que algunas ventajas de ese lapso del pasado -y que nos es de gran utilidad para debatirlas en torno a la historia enseñada- son: a) delimita un lapso temporal más o menos acotado; b) replantea la relación sujeto-objeto, en tanto el recuerdo puede corresponder o no a la generación a la que pertenece el historiador; c) delimita el presente histórico a un marco temporal de sentido determinado por la intersección de los espacios de experiencia de las 3 generaciones.

En el afán de establecer una nueva periodización, encontramos diversidad de acontecimientos que delimitarían esta época: la Segunda Guerra Mundial (CUESTA BUSTILLOS, 1998); la Revolución cultural de los años 1960 (AGUIRRE ROJAS, 2000); los años 1990; historia de la gente viva del mundo actual (ARÓSTEGUI; BUCHRUCKER; SABORIDO, 2001); cambio en el capitalismo y sus manifestaciones nacionales (FIGUEROA; IÑIGO, 2010); 1989, un momento como idea de ruptura, cambio radical y apertura a nueva época (TRAVERSO, 2010); los procesos de democratización (NOVARO; PALERMO; 2004); y la crisis del 2001 (ANSALDI, 2003), entre otros.

Lo que hoy podemos sostener es que tanto la historia reciente como presente, dependiendo de la comunidad de cientistas sociales que se aboquen a este tiempo histórico, ha cobrado un impulso significativo en el ámbito 
académico, en el que el parámetro móvil de esta temporalidad se construye a partir de la diversidad de problemas objeto de estudio. Franco y Lvovich (2017) plantean que este tipo de historiografía surge en países que han atravesado situaciones de violencia social o estatal cuyas demandas de justicia aún siguen pendientes. En este sentido, la autora y el autor sostienen que "la historia reciente refiere a procesos históricos cuyas consecuencias directas conservan aun fuertes efectos sobre el presente, en particular en áreas muy sensibles, como el avasallamiento de los derechos humanos más elementales" (FRANCO; LVOVICH, 2017, p. 191).

Las incertidumbres planteadas en este debate nos dan ciertas certezas para confirmar que la historia de esta época no se entiende como una época determinada, con una delimitación temporal estática y fija, sino como una categoría dinámica e inacabada. La ausencia de hitos cronológicos fijos que la delimiten nos indica que estamos frente a procesos, dinámicos, abiertos e indeterminados. Se tratan de parámetros temporales móviles y flexibles que articulan fenómenos y acontecimientos que han sucedido hace poco tiempo historia reciente- con aquellos que suceden mientras se escribe o enseña esta historia -historia presente-. Estamos ante una HRP que se presenta potente para configurar propuestas de enseñanza.

La HRP, entonces, posibilita un análisis histórico de la dinámica de la realidad social en tanto comporta una relación de coeternidad entre la historia vivida y la escritura de esa misma historia, entre los actores, testigos y especialistas académicos de esos procesos históricos. La HRP es la expresión de una relación compleja de temporalidades múltiples, en la que se pueden superar los estrechos límites del tiempo corto y prolongar su análisis en la larga duración. Se trata de un tiempo proceso que está siendo en el que se entretejen, a partir de un problema, componentes de tiempos, sujetos y territorialidades múltiples y diversas.

Desde esta perspectiva, claro está, no se da una solución epistemológica a la complejidad del debate caracterizado; muy por el contrario, se trata solo de un enfoque didáctico que posibilitaría un andamiaje entre el origen del problema y el tiempo de duración del mismo, en el que la decisión sobre una temporalidad 
móvil y sus operadores estará orientada por las finalidades que el profesorado defina y por las evidencias que permitan pensar el problema.

Esta construcción y el recorrido (acotado en este texto) fue posible considerando los resultados de nuestras investigaciones, fundamentalmente las desarrolladas durante los años 2006 y 2012. El Proyecto de Investigación (PI) C068 2006-2009 nos ofreció una serie de datos e información que nos posibilitó conocer qué historia se enseña en la escuela y sobre de la presencia o ausencia de la HRP en las prácticas del profesorado. Por ejemplo, con relación a los contenidos de la HRP, el 7\% -de 60 profesoras y profesores encuestados ${ }^{2}$ - no los incluye en su programación, un 33\% manifiesta que no acuerda con ellos por su carácter controversial, subjetivo o desconocimiento del avance historiográfico sobre los mismos. El profesorado que sí los incluye suma un 42\% y los piensa como eje de la enseñanza, y el 12\% restante indica que lo trabaja en la última unidad de la programación, lo que presupone una perspectiva temporal progresiva a la cual, por cuestiones de tiempo -dada la extensa cantidad de contenidos organizados en la planificación anual-, escasamente se llegan a abordar en la clase. Sin embargo, en su conjunto, el 70\% manifiesta que estos contenidos no ofrecen dificultades en el proceso de enseñanza.

Estos datos cuantitativos, al triangularse con los que emergieron de los 15 estudios de casos realizados, nos indican que, por un lado, el abordaje de enseñanza de la HRP no resulta indiferente al profesorado, muy por el contrario, plantean que es importante y necesario incorporarlo en la enseñanza. Por otro, que las finalidades en la enseñanza de la HRP posibilitan que las jóvenes generaciones se acerquen a: la comprensión/explicación de los contextos actuales nacional y mundial; la socialización política a partir del tratamiento de temáticas centradas en la ciudadanía; la articulación entre enseñanzas, historias, identidades y memorias; y para educar en la temporalidad y en la conciencia histórica.

\footnotetext{
El cuestionario se organizó sobre los siguientes ejes, lo que nos permitió recabar y analizar los datos: Formación, Antigüedad, Incidencia de la Capacitación, Incidencia de Aspectos Institucionales, Estrategias, Recursos y Valoración de la Enseñanza de la HRP. Población joven con pocos años de antigüedad en la docencia y, mayoritariamente, 82\% egresados de la universidad.
} 
En consonancia con los debates historiográficos, según el dato obtenido de las encuestas, un porcentaje importante (40\%) del profesorado no los considera para organizar sus propuestas de enseñanza aunque, paradójicamente, en las entrevistas en profundidad y del análisis de documentos proporcionados por el profesorado hemos podido constatar que sí se plantean, como finalidades de la historia escolar, algunos de los núcleos de las discusiones historiográficas, por ejemplo: la relación de la democracia y la ciudadanía como una cultura política característica de la HRP a partir de ejemplos, comparaciones o contranarrativas de los procesos que enseñan; la educación temporal compleja relacionada al desarrollo de la conciencia histórica que resitúa a los sujetos en perspectiva del tiempo futuro como construcción humana; la incorporación de PS en la HRP vinculados a procesos traumáticos como lo es el Golpe de Estado en 1976 o la crisis del 2001 en Argentina; y aquellas cuestiones vinculadas a la memoria e identidad en el proceso de democratización de la sociedad a partir de 1983. En definitiva, la HRP interesa y preocupa al profesorado, sin embargo, la matriz de una historia positivista se encuentra latente y residual a la vez, sumada a las carencias curriculares y de formación sobre esta problemática-cuestiones estas que nos aventuraron a la producción de material didáctico y curricular acompañado de formación situada, permanente y colaborativa con el profesorado-. Actividad que realizamos en cada uno de los PI que emprendemos.

El segundo PI -C089 2010-2012 "Profesores y enseñanza de la HRP”se configuró como problema y objeto de investigación a partir de los resultados obtenidos en el PI anterior. Con un proceso metodológico cualitativo, critico, participativo y colaborativo, nos abocamos a estudiar casos concretos y a la construcción de dispositivos pedagógicos y didácticos para la enseñanza de la HRP en escuelas secundarias y primarias de la región, desde una perspectiva colaborativa y participativa con el profesorado. Nos planteamos analizar la relación entre contenidos y formas de la enseñanza de la HRP que despliegan docentes; indagar los significados de la HRP que sostienen los docentes; establecer las relaciones entre la enseñanza de la HRP y la formación de la historicidad y de la conciencia histórica en los jóvenes; y establecer relaciones entre la enseñanza de la HRP y la construcción de identidades y memorias. 
La historia reciente/presente desde una perspectiva de los problemas sociales en la educación de ciudadanías

Como anticipamos, la denominación y demarcación de un campo de conocimiento, como lo es la HRP, implica reconocer la articulación de disciplinas, metodologías, fuentes, perspectivas políticas educativas y filosóficas, en definitiva, la multiplicidad de componentes de un complejo campo que procura conocer, producir y comunicar procesos históricos. Esta complejidad no está ausente en el pensamiento del profesorado con el que hemos trabajado, de hecho, plantean diversas concepciones y valoraciones historiográficas que orientan las decisiones en sus prácticas. Sin embargo, no identificamos conocimiento profundo sobre la articulación entre historiografía y enseñanza de la HRP. El esfuerzo intelectual del profesorado, que tiene un claro posicionamiento con relación a la importancia de incorporar la HRP en sus propuestas, se nutre de diversas fuentes de información, aportes interdisciplinares y recursos y materiales didácticos que de forma creativa -ante la escasez de materiales curriculares con un tratamiento académico de la HRPutiliza para producir conocimiento escolar y provocar aprendizajes críticos en el estudiantado.

El campo diverso de nuestras investigaciones dialoga con experiencias, perspectivas, representaciones y posicionamientos igualmente diversos. Esta diversidad se manifiesta en las clases de historia y en la construcción de los dispositivos colaborativamente construidos con el profesorado sobre algunos de los contenidos de la HRP3. La nota distintiva, que contribuye a pensar epistemológicamente la HRP, según el profesorado, se centra en el tratamiento de los PS del hoy, con los ojos puestos en el futuro, sin desconocer su historicidad.

\footnotetext{
Los dispositivos construidos colaborativamente, en un espacio de auto-co-formación que fueron implementados en secuencias didácticas y luego evaluados para reconstruirlos y socializarlos como material didáctico giraron en torno, entre otros, a los siguientes problemas: "La ciudadanía y las problemáticas sociales y ambientales"; "Los conflictos socio políticos y de la participación ciudadana durante la crisis del 2001"; "Los gobiernos democráticos de Argentina desde 1983 hasta la actualidad"; "Democracia/dictadura hasta la crisis de la globalización: las violaciones de los DD.HH”; "La difícil relación entre capital-trabajo-Estado. El peronismo como paradigma político"; "La emergencia de los nuevos movimientos sociales en América Latina"; "Resistencias, rebeliones y protestas sociales durante el s. XX y XXI en Argentina: la profundización de la violencia estatal, institucional y social durante las décadas del 60 y 70 en Argentina (1966-1973)".
} 


\section{Las finalidades y la educación ciudadana en historia reciente/presente}

La EC ha sido una preocupación no solo en nuestro país, sino en diversos rincones de este mundo global. Por ejemplo, para el ámbito europeo, el español Ernesto Gómez Rodríguez (2004) sostiene que la ciudadanía, en las últimas 2 décadas, ha sido el foco de interés en diversas disciplinas y perspectivas teóricas, pero, fundamentalmente, por el impacto que han tenido determinadas transformaciones producidas en las sociedades contemporáneas. El autor reconoce 2 grandes procesos de cambio que han ido configurando otra idea de ciudadanía; en primer lugar, a aquellos cambios se son consecuencias del fenómeno de la globalización que, entre otras dimensiones, se ve reflejado en:

[...] la creciente interdependencia de las economías nacionales, la formación de un mercado de trabajo mundial, el desarrollo de nuevos centros económicos junto con la desaparición de los viejos centros tradicionales, están deviniendo en fenómenos cotidianos que a su vez dan lugar a profundos cambios en el ámbito de la ciudadanía (GOMEZ RODRIGUEZ, 2004, p. 1-2).

Uno de los efectos del proceso de interdependencia económica, para el autor, se traduce en el auge de los movimientos migratorios y el consecuente problema del crecimiento de una ciudadanía formal, en los procesos de una identidad ciudadana que vira hacia una definición multicultural de la misma, a la integración de nuevas unidades políticas y económicas supranacionales, con las consecuentes implicaciones jurídicas y de políticas públicas que, paradójicamente, tienen unos efectos contrarios a los esperado.

El segundo proceso al que refiere Gómez Rodríguez (2004) se inscribe en el avance de las políticas neoliberales para desmantelar el Estado de bienestar y ello se constata en que:

La revisión neoliberal va acompañada de una serie de consideraciones sobre la ciudadanía y una de ellas versa sobre las consecuencias que se derivan de la reestructuración de los mercados de trabajo sobre los derechos de ciudadanía; más concretamente, exigen analizar el impacto que la emigración que reciben los países más desarrollados tiene sobre las transformaciones o involuciones de algunos derechos sociales fundamentales de sus ciudadanos (GOMEZ RODRIGUEZ, 2004, p. 2-3). 
Las políticas neoliberales, además, insiste el autor, han producido nuevas desigualdades sociales y ello se ve reflejado en los nuevos movimientos sociales relacionados con las etnias, el género, la edad, la cultura. En definitiva, el neoliberalismo ha configurado un escenario económico, social, político y cultural de alcance global en el que las ciudadanías se redefinen en el marco de unas realidades complejas, pero con matices específicos de acuerdo a los procesos históricos, socio-políticos y culturales concretos.

En nuestra sociedad, pos dictadura, la democracia irrumpe como un imperativo que requiere ser profundizada desde políticas públicas claras. La educación es un instrumento fundamental, porque como sociedad carecíamos de la posibilidad de prácticas democráticas. Se trata de un proceso abierto que debe orientarse sobre valores humanos irrenunciables, pero también sobre las nuevas prácticas de la ciudadanía que se traduce en diversidad de movimientos sociales que pujan por un espacio de visibilidad en la escena pública y más derechos que igualen.

En el ámbito educativo, a comienzos del retorno de la democracia en Argentina, hemos asistido a profundas discusiones (SIEDE, 2007) que van de posicionamientos políticos en cuanto a quién o quienes se atribuyen la formación de niñas, niños, jóvenes y adultos en determinados valores, pasando por la diversidad de denominaciones que tendría la asignatura en el currículo hasta el detalle cuidadoso de los contenidos que deberían incluirse en el mismo. Una discusión que trascendió el ámbito escolar y se instaló como un problema social, cultural y epistemológico. En el vaivén de las disputas sectoriales -Estado, familias, Iglesia, corporaciones, sociedad civil, medios de comunicación-, la enseñanza de los PS que afectan a las ciudadanías se introducían progresivamente en las prácticas del profesorado como una necesidad para ofrecer oportunidades a un estudiantado curioso de la dinámica social de la realidad.

La EC no será un espacio de contenidos renovados, sino hasta comienzo de este milenio. La formación cívica centrada en el conocimiento de derechos civiles, sociales y políticos viró en resignificar la democracia, los derechos humanos y la participación ciudadana como principios indiscutibles en la 
formación de las futuras generaciones. En este proceso, la HRP se va consolidando como enfoque actualizado desde perspectivas que echan luz sobre los PS que requieren ser pensados históricamente.

A comienzos de este siglo, las perspectivas y concepciones sobre las ciudadanías recobran fuerza y ello se deja entrever en las normativas educativas a nivel nacional y, fundamentalmente, en los currículums elaborados, en cada jurisdicción, a parir de la última Ley Nacional de Educación del año 2006 (JARA; FUNES, 2018). En las propuestas curriculares, la EC se consolida como una asignatura -con diferentes denominaciones- y un espacio de articulación interdisciplinar en el área de las ciencias sociales y humanas, de la escuela secundaria, con contenidos variados, pero con énfasis en los procesos contemporáneos, en los que perspectivas tradicionales se conjugan con nuevas miradas y reconocimientos de las ciudadanías del siglo XXI (JARA, 2020).

Entre la diversidad de temas/problemas que se incorporan para la EC se destacan la democracia, los derechos humanos, la interculturalidad, el género, los pueblos originarios, los nuevos movimientos sociales, problemas socio ambientales, migraciones, economía social, y el fracking, entre otros tantos que procuran la formación de ciudadanías críticas y participativas para la resolución de los PS glocales. Las finalidades que se persiguen tienen como horizonte aportar conocimientos que promuevan un pensamiento comprensivo y crítico del complejo mundo actual. La escuela sigue siendo un lugar necesario y esencial para democratizar el conocimiento, respetar la diversidad y la pluralidad de pensamientos y contra-argumentar a los defensores del pensamiento único. Socializar y contrasocializar son acciones complementarias en la EC.

Mientras que la socialización aporta los elementos integradores del individuo en la sociedad, la contrasocialización aporta los elementos analítico críticos que le permitan considerar y juzgar la sociedad en la que se integra [...] la contrasocialización es un pilar básico de la educación democrática, ya que contribuye a estimular el pensamiento crítico del alumnado, permitiéndole analizar, reconocer y valorar los comportamientos sociales propios y ajenos (GOMEZ RODRIGUEZ, 2008, p. 135).

En sociedades con democracias jóvenes, como la nuestra, resulta esencial educar para fortalecer y ensanchar los márgenes restrictivos de una democracia 
nominal o de baja intensidad. Articular en la enseñanza de la HRP los PS es un desafío para la EC en tanto que, como plantea Funes (2011), una formación democrática se afirma en el reconocimiento de la pluralidad de culturas e identidades. Participar, asumir un punto de vista, argumentar en el debate público, se torna importante en el desarrollo de una práctica ciudadana.

En el caso de la HRP de Argentina, algunos acontecimientos se constituyen en citas inevitables para abordar la experiencia como un campo socio-político que fue contestatario en la vasta movilización política y social de las décadas de los 60 y 70; traumático en la nefasta dictadura de 1976; de erosión social en la hiperinflación de 1989; de profunda exclusión en la crisis del 2001. Fueron clivajes que erosionan cualquier referente de certidumbre respecto del futuro, se constituyen en acontecimientos y procesos urticantes y sensibles que ofrecen lecturas contrapuestas. Los argentinos vivimos en una sociedad en permanente conflicto que está muy lejos de ser una sociedad conciliada. La naturaleza de los antagonismos que atraviesan nuestra sociedad es de lo más variada: conflictos políticos, económicos, sociales, étnicos, culturales. La desigualdad social -visible en grados extremos en una sociedad democrática- aparece como la divergencia de más difícil resolución para el establecimiento de un orden estable (FUNES, 2011, p. 59).

Finalmente, queda por destacar que, en la enseñanza de la HRP a partir de PS, las finalidades claras son fundamentales para organizar una propuesta que se plante comprender la realidad social, formar un pensamiento crítico y creativo e intervenir socialmente, y transformar la realidad (SANTISTEBAN, 2011).

\section{Los problemas sociales como contenido en la educación histórica de las ciudadanías}

¿Qué ciudadanía para el siglo XXI?

Esta es una pregunta que se ha instalado en la agenda educativa y respuestas a ella encontramos en diversidad de producciones e investigaciones en el campo de la didáctica de las ciencias sociales y de la historia y también en otros ámbitos, como el teórico, el educativos, o en el de las políticas públicas, por ejemplo. La preocupación en torno a la pregunta no es nueva, aunque, desde las respuestas epistemológicas construidas y de las prácticas situadas de 
enseñanza, se han ido configurando unos contenidos actualizados en el marco de la complejidad del mundo actual.

En el campo educativo, la pregunta remite al contexto, los actores, las finalidades, y al contenido que intervienen en el proceso de la enseñanza de la HRP. En países como el nuestro, la importancia de fortalecer la democracia se constituye en el horizonte de la formación ciudadana. En la Argentina de pos dictadura, a partir de 1983, se abre un proceso de democratización de la sociedad, la cultura y las instituciones, entre ellas la escuela es quien tendrá una de las funciones centrales en este proceso. Se trata de formar a las futuras generaciones en principios y valores democráticos sostenidos en los derechos humanos y en el conocimiento de los procesos de la HRP para generar una conciencia ciudadana del "nunca más". Desde la educación, no se plantea la solución a la crisis estructural provocada por el terrorismo de Estado -1976-, ni de los problemas cotidianos que viven las personas, sino de formar ciudadanías que se sientan protagonistas, desde la participación política, en la construcción de futuros democráticos, inclusivos y plurales.

En Argentina, la EC ha ido virando -en su denominación, carga horaria, finalidades, y contenidos- desde una perspectiva que planteaba a este espacio a partir de la transversalidad a la articulación y el dialogo de saberes, al menos en el campo de las ciencias sociales y humanas. También hemos pasado de la formación centrada en el civismo, con énfasis de un enfoque jurídico y político derechos de primera y segunda generación, conocimiento del ordenamiento, y funcionamiento de las instituciones del Estado- a la incorporación de los cambios culturales y socio-políticos de la HRP. En este proceso de discusiones políticas y epistemológicas, hemos realizado un avance significativo para pensar en la formación ciudadanías democráticas en un mundo dinámico y cambiante que necesita construir argumentos complejos porque la realidad no se presenta lineal ni unicausal.

La EC como asignatura escolar, en nuestro país, ha sido uno de los espacios curriculares que han introducido los problemas de la ciudadanía, la democracia, los derechos humanos, la interculturalidad, entre otros, como objeto de estudio y de análisis para la construcción de una cultura política y una cultura 
cívica de participación ciudadana (JARA y FUNES, 2016). Como espacio en el currículum, no estuvo exento de tensiones y posicionamientos encontrados, porque se trata de una asignatura con profundas connotaciones políticas y en un inicio se ha puesto en tela de juicio -por algunos sectores sociales como padres, autoridades e incluso el profesorado- que podría tratarse de una asignatura de política partidaria o que aborde temas que quedan resguardados a la intimidad y las creencias familiares.

En este marco, en Argentina se sancionaron 2 leyes de educación ${ }^{4}$ que han establecido principios y orientaciones generales para la formación de ciudadanías comprometidas con los valores éticos y democráticos de participación. Normativa que se constituyó en basamento para la elaboración de diseños curriculares en todo el país y han sugerido la incorporación de perspectivas y enfoques actualizados desde las ciencias sociales y humanas, para organizar contenidos escolares.

Entre la diversidad de temas que se incorporaron al currículo y a las prácticas de enseñanza, los socialmente controvertidos han tenido un tratamiento diverso entre el profesorado y un interés particular en el estudiantado. En relación al profesorado, nuestras investigaciones nos indican que la formación inicial ha sido escasa y hasta nula con relación al conocimiento de la EC, lo que conlleva cierta dificultad en su tratamiento, aun así, una parte del profesorado indica que aborda problemas o conflictos sociales candentes en sus clases y otro tanto manifiesta que algunos de los temas son muy controversiales, si se consideran las experiencias vitales de algunos estudiantes y las familias; como, por ejemplo, el accionar de la policía o de las fuerzas armadas durante la última dictadura, la violación sistemática de los derechos humanos, la violencia de género, o las políticas públicas con relación a la inmigración, entre otros.

Desde el punto de vista del estudiantado, los problemas que cotidianamente experimentan en sus comunidades y a nivel glocal, son de interés y preocupación. Se involucran en el tratamiento de temas relacionados con la

\footnotetext{
${ }^{4}$ La Ley Federal de Educación (LFE) - Ley No. 21.195 del año 1993-y la Ley de Educación Nacional
} (LEN) -Ley No. 26.206 del año 2006-. Ambas sancionadas durante la vida democrática del país. 
discriminación, la violencia, el prejuicio, las desigualdades económicas y políticas, la pobreza, la alteridad, y la resolución de conflictos en los procesos de la HRP. En general, tienen un posicionamiento frente a algunas de las temáticas, aunque, mayoritariamente, reflejan representaciones y valoraciones construidas en otros espacios de socialización -como los medios de comunicación tradicionales y los de mayor utilización, como lo son las redes sociales- con escasos o imprecisos argumentos epistemológicos que la escuela les pudiera haber ofrecido para leer la dinámica de la realidad social. Aspectos que hemos podido construir con el desarrollo del PI C106 -“Enseñanza y aprendizaje de las ciencias sociales y de la historia en la cultura digital” (2013-2016) - y del PI C129 -“El aprendizaje escolar de las ciencias sociales en la cultura digital" (2017-2020)-.

El panorama de la EC en Argentina es amplio, dinámico, y heterogéneo. Hasta hace poco tiempo ${ }^{5}$, en muchos de los casos quedaba sujeto a las decisiones profesorales o de alguna institución educativa concreta, abordar y/o tratar PS específicos. Hoy podríamos decir que la formación política y ciudadana tiene un espacio específico en el currículo, lo que no se traduce linealmente a las prácticas de enseñanza.

En el marco de nuestras investigaciones y a partir de la diversidad de dificultades que nos ha planteado el profesorado vinculadas con la escasa formación recibida para tratar la complejidad de la EC en la escuela, hemos desarrollado diversidad de propuestas de enseñanza para abordar los temas relacionados a los procesos de la HRP. En general, hemos elaborado propuestas ${ }^{6}$ enfatizando en la actualización y renovación epistemológica en el campo de las ciencias sociales con orientaciones didácticas para abordar PS candentes y relevantes en las clases de ciencias sociales, historia y geografía. Nuestras propuestas se han nutrido de las investigaciones, las actividades de formación inicial y continuada, y han tenido como destinatarios al profesorado de las escuelas de nuestra región.

\footnotetext{
5 Desde el año 2006, con la LEN, el proceso de reformas curriculares en todo el territorio se ha producido en diversos tiempos. En la provincia de Río Negro, por ejemplo, el Diseño Curricular fue aprobado en el año 2017 y en la Provincia del Neuquén en el año 2018; para ambos casos, la formación política y ciudadana se incorpora como asignaturas y talleres en la educación de adolescentes y jóvenes de la escuela secundaria, en el área de las ciencias sociales y humanas.

${ }^{6}$ El material didáctico está disponible en www.apehun.uncoma.edu.ar.
} 
Los temas/problemas objeto de estudio siempre estuvieron orientados por las finalidades que el profesorado se plantea como desafíos, de allí que los contendidos contemplen algunos de los diversos aspectos y componentes de una realidad social dinámica. Hemos compartido, con el profesorado que cotidianamente enseña en la escuela secundaria, que la enseñanza de la historia, de la geografía, o la EC siempre se ha relacionado con la finalidad de una EC. El debate de ideas, significados, y propuestas ha tenido como horizonte un tópico común: formar ciudadanías críticas, con autonomía de pensamientos argumentados, que le posibiliten participar y tomar decisiones en el mundo que habitan.

Lo recuperado de nuestras investigaciones evidencia la actualidad, la persistencia, y el desafío de un tema/problema que invita a continuar profundizando el conocimiento y la enseñanza de la HRP, atendiendo a problemas sensibles y controversiales que caracterizan el mundo global que habitamos, en los que, sin dudas, las injusticias, la marginalidad, la pobreza, la migración forzada, la contaminación ambiental, los femicidios, la desocupación, las prácticas políticas autoritarias, entre tantas otras, son problemas glocales que ameritan un tratamiento contextualizado de manera que ofrezcan al estudiantado la posibilidad empática para construir colectivamente otros futuros inclusivos. Es necesario ofrecer una educación, como planteaba el maestro Paulo Freire, que cambie a las personas que van a cambiar el mundo.

\section{Referencias}

AGUIRRE ROJAS, Carlos Antonio. Pensamiento historiográfico e historiografía en el Siglo XX: ensayos introductorios. Rosario: Prohistoria, 2000.

ANSALDI, Waldo. Democracias de pobres, democracias pobres, pobres democracias. Temas y debates, Rosario, Año 7, n. 6/7, p. 27-43, 2003

ARÒSTEGUI, Julio. La idea de una historia del presente. Madrid: Universidad Complutense (Curso de Doctorado 1997-1998), 1997. Mimeografiado.

ARÒSTEGUI, Julio. Tiempo contemporáneo y tiempo presente: una reconsideración necesaria. In: DÍAZ BORRADO, Mario (coord.). Historia del tiempo presente: teoría y metodología. Salamanca: I.C.E Universidad de Extremadura, 1998. p. $41-75$. 
AROSTEGUI, Julio; BUCHRUCKER, Cristian; SABORIDO, Jorge. El mundo contemporáneo: historia y problemas. Buenos Aires Biblos, 2001. p. 67-89.

AUTOR; FUNES, Alicia Graciela. ¿Qué ciudadanía? orientaciones normativas y curriculares en Argentina. Ensino Em Re-Vista, Uberlandia, v. 25, p. 917-936, jun./dic. 2018. Número especial.

AUTOR; FUNES, Alicia Graciela. Didáctica de las ciencias sociales desde una perspectiva y enfoque de la educación ciudadana. In: AUTOR; FUNES, Alicia Graciela (comp.). Didáctica de las ciencias sociales en la formación del profesorado: perspectivas y enfoques actuales. Cipolletti: Ed. UNCo, 2016. p. 3162.

AUTOR; FUNES, Alicia Graciela. Enseñanzas de historias recientes/presentes y la educación para las ciudadanías. Revista de Investigación en Didáctica de las Ciencias Sociales (REIDICS), Extremadura, n. 7, p. 30-44, jul./dic. 2020.

AUTOR. El enfoque interdisciplinar en la enseñanza de las ciencias sociales y humanas. reflexiones epistemológicas y metodológicas. Revista Clío \&Asociados: La Historia enseñada, Santa Fe: UNL y UNLP, n. 30, p. 75-89, mar./jun. 2020.

CUESTA BUSTILLO, Josefina. Memoria e historia. Madrid: Ed. Marcial Pons, 1998.

FRANCO, Marina; LVOVICH, Daniel. Historia reciente: apuntes sobre un campo de investigación en expansión. Boletín del Instituto de Historia Argentina y Americana “Dr. Emilio Ravignani”, Buenos Aires, n. 47, p. 190-217, 2. sem. 2017. Tercera serie.

FUNES, Alicia Graciela; AUTOR. El pasado reciente en las prácticas de enseñanza de las ciencias sociales y la historia. In: PLÁ, Sebastián; RODRÍGUEZ ÁVILA, Sandra (coords.). Saberes sociales para la justicia: educación y escuela en América Latina. Bogotà: Ed. La carreta: Universidad Pedagógica Nacional, 2017. 45-70.

FUNES, Alicia Graciela; JARA, Miguel Angel. La enseñanza de las ciencias sociales, la historia, la geografía y los problemas sociales. In: FUNES, Alicia Graciela; JARA, Miguel Angel (comp.). Historia y geografía, propuestas de enseñanza. Cipolletti: EDUCO, 2015. p. 13-32.

FUNES, Alicia Graciela. La enseñanza de la historia y los problemas sociopolíticos: de la historia reciente al futuro. In: PAGÉS, Joan; SANTISTEBAN, Antoni (comp.). Les questions socialment vives i lenseyament de les ciencias socials. Barcelona: Documents 97, 2011. p. 53-63. 
GÒMEZ RODRIGUEZ, Antonio Ernesto. Ciudadanía y enseñanza de las ciencias sociales. In: VERA MUÑOZ, María Isabel; PÉREZ I PÉREZ, David (coord.).

Formación de la ciudadanía: nuevos problemas y nuevas técnicas de información y comunicación. Alicante: Universidad de Alicante: AUPDCS, 2004. p. $1-11$.

GÒMEZ RODRIGUEZ, Antonio Ernesto. Educación para la ciudadanía: una aproximación al estado de la cuestión. Enseñanza de las Ciencias Sociales, Barcelona, n. 7, p. 131-140, 2008.

MUDROVCIC, María Inés. Algunas consideraciones epistemológicas para una historia del presente. Hispania Nova Revista de Historia Contemporánea, Madrid, v. 1, n.1, p. 1-13, 2000. Disponible en: https://erevistas.uc3m.es/index.php/HISPNOV/index. Acceso en: 25 ago. 2021

PAGĖS, Joan. La educación para la ciudadanía y la enseñanza de la historia: cuando el futuro es la finalidad de la enseñanza del pasado: reseñas de enseñanza de la historia. Apehun/Alejandría, Córdoba, n. 6, p. 71-89, sept. 2008.

SANTISTEBAN FERNANDEZ, Antoni. La enseñanza de las ciencias sociales a partir de problemas sociales o temas controvertidos: estado de la cuestión y resultados de una investigación. El Futuro del Pasado, Salamanca, n. 10, p. 5779, 2019.

SANTISTEBAN FERNANDEZ, Antoni. Las finalidades de la enseñanza de las ciencias sociales. In: SANTISTEBAN, Antoni; PAGĖS BLANCH, Joan (coords.). Didáctica del conocimiento del medio social y cultural en la educación primaria. Madrid: Síntesis, 2011. p. 63-84.

SIEDE, Isabelino. La educación política: ensayos sobre ética y ciudadanía en la escuela. Buenos Aires: Paidós, 2007

TRAVERSO, Enzo. Discurso introductorio. In: JORNADAS DE TRABAJO SOBRE HISTORIA RECIENTE, 5., 2010. Buenos Aires. Actas [...]. Buenos Aires: Universidad Nacional General Sarmiento, 2010. p. 1-5. 